\title{
Peer-reviewed research
}

\section{Did Bubble Activity Intensify During COVID-19?}

\author{
Paresh Kumar Narayan ${ }^{1}$ a \\ 1 Deakin Business School, Deakin university, Australia \\ Keywords: covid-19, exchange rates, bubbles \\ https://doi.org/10.46557/001c.17654
}

\section{Asian Economics Letters}

Vol. 1, Issue 2, 2020

In this note, we utilize hourly exchange rate data for Japanese Yen, Canadian dollar, European Euro and the British pound to search for possible bubble type behavior. We identify evidence that bubble activity characterizes all four exchange rates more so in the COVID-19 period. We also show that bubble activity intensified during the COVID-19 period, implying markets became relatively more inefficient compared to the pre-COVID-19 period.

\section{Introduction}

Searching for bubble activity or bubble type behavior in asset prices has occupied historical interest, beginning with the Dutch tulipmania in 1634-1637 (Garber, 1989). Bubbles have been documented in recent work; such as, inter alia, Phillips et al. (2011), Phillips et al. (2015), Bettendorf \& Chen (2013), Narayan et al. (2016) and Hu \& Oxley (2017). The message is that bubbles and crises have a direct link and the literature on bubbles has been motivated by extreme events or crises. The presence of bubbles in prices indicates market inefficiency-from a theory point of view, evidence on bubbles allows a test of the market efficiency hypothesis.

The subject of this note is no different. Inspired by the ramifications of COVID-19 (see Sha \& Sharma, 2020), we ask whether bubble activity in exchange rates has intensified in the COVID-19 period. We use the Phillips et al. (2011) bubbles test model (described in Section II) to examine bubble type behavior in four exchange rates, namely, the Canadian dollar (CAD), the Japanese Yen (YEN), the European Euro (EURO), and the British pound (GBP). Our main finding is that overall bubble activity intensified in the period marked by the COVID-19 pandemic. In the preCOVID-19 period (July 2019 to Dec 2019) based on hourly exchange rate data, we find the number of hours of bubbles to be 80, 97, 99 and 115 for CAD, YEN, EURO, and GBP, respectively. The corresponding number of bubbles in the COVID-19 sample (January 2020 to September 2020) was $101,241,134$, and 170 hours. This implies that bubble activity in the exchange rate market increased in all markets in the COVID-19 period and it more than doubled in the case of the YEN. The implication is clear: exchange rate markets became inefficient in the COVID-19 period.

This note, by exploring exchange rate bubble activity, sets the agenda for future research to expand on this theme. To this effect, given the preliminary nature of our work and constrained by space in a letters journal, there are several limitations of our work. First, we leave robustness testing to future research. Second, while we utilize single bubbles test there are also multiple bubbles test, such as the generalized sup augmented Dickey-Fuller (ADF, 1979) test, which is essentially a rolling version of the sup ADF test (see Phillips et al., 2015) . Future studies can explore this too. Our point is that if one-bubble activity is intense in the COVID-19 period, the two-bubble test will be even stronger. We leave this for future studies to verify. Third, our empirical work is rooted in the market efficiency theory. While the existence of greater bubble activity implies that exchange rate markets became inefficient during the COVID-19 period, we do not engage in trading strategies in the exchange rate market. We leave this for future studies.

In closing, we highlight that there is scarce literature on the exchange rate-COVID-19 nexus despite a large and growing literature on COVID-19 (see Al-Awadhi et al., 2020; Devpura \& Narayan, 2020; Ertuğrul et al., 2020; Fu \& Shen, 2020; Gil-Alana \& Monge, 2020; Gu et al., 2020; Haroon \& Rizvi, 2020a, 2020b; Huang \& Zheng, 2020; Iyke, 2020a, 2020b; Liu et al., 2020; Narayan, 2020; Qin et al., 2020; A. Salisu \& Adediran, 2020; A.A. Salisu et al., 2020; Afees A. Salisu \& Vo, 2020; Xiong et al., 2020; Zaremba et al., 2020; Zhang et al., 2020). In this literature, we come across only two studies that explore the dynamics of exchange rates visà-vis the pandemic. Narayan et al. (2020) show that the Yen predicted the Japanese stock market returns more strongly over the COVID-19 period, suggesting that the information content in the Yen was richer during the pandemic. We contribute by showing greater bubble activity which can be perceived as a source of information during the pandemic. Iyke (2020a) show that COVID-19 predicts exchange rates, suggesting like in our study that there is a relation between the pandemic and the exchange rate.

Finally, our work and findings are consistent with those on exchange rate bubbles. In this regard, Hu \& Oxley (2017) find mixed evidence of bubbles in a large number of currencies while Bettendorf \& Chen (2013) find explosive behavior in the GBP but argue that this maybe driven by relative prices of traded goods such that GBP may not be char-

a Corresponding author: Centre for Financial Econometrics, Deakin University. Email: narayan@deakin.edu.au

Helpful comments and suggestions from an anonymous reviewer of this journal helped improve the paper. The usual disclaimer applies. The research is this paper is supported by funding obtained from the Centre for Financial Econometrics at Deakin University, Melbourne, Australia. 
acterized by rational bubbles. We differ on two fronts: first, we use high frequency (hourly data) as opposed to monthly data predominantly used by the literature; and, second, we consider the COVID-19 pandemic data.

We discuss the data, model and results in the next section. A summary is provided in Section III as a conclusion to this note.

\section{A test of bubbles in exchange rate}

\section{A. Model}

Our approach to computing bubbles follows a recent econometric procedure developed by Phillips et al. (2011). The testing procedure is based on the familiar ADF regression model, which is of the following form:

$$
\begin{aligned}
\Delta E R_{t}= & \pi+\rho E R_{t-1}+\sum_{j=1}^{k} \psi_{j} \Delta E R_{t-j} \\
& +\mu_{t}, \mu_{t} \sim N I D\left(0, \sigma^{2}\right)
\end{aligned}
$$

In this model, $E R$ is one of the four exchange rates we have. We have a dataset that is sampled hourly. We have a 17-hour day, starting at 1:00am and ending at 5:00pm. The data sample is from 01/07/2019: 2:00am to 04/09/2020: 5:00pm. We source all data from REFINITIVE, Datascope. The fullsample of data is split into a pre-COVID-19 (01/07/2019: 2:00am to 31/12/2019: 5:00pm) and the COVID-19 sample (1/01/2020: 1:00am to 04/09/2-2-: 5:00pm). The optimal lag length, $k$, is Schwarz Information Criterion based. The null hypothesis is that there is a unit root, which is tested as $H_{0}: \rho=0$ against the alternative hypothesis $H_{1}>0$, suggesting an explosive root. Phillips et al. (2011) propose estimating the ADF model using least squares. Our approach is the same as the recursive regression procedure, whereby the ADF model is estimated recursively using subsets of the sample data. The corresponding $t$-test statistics are generated following the procedure outlined in Phillips et al. (2011). We set the initial window to 100 hours. The critical values used for testing the null hypothesis are given by $\mathrm{CV}(s)=\frac{\log (\log (\mathrm{ns}))}{100}$. By generating these $t$-statistics and comparing them with the critical value, we obtain the number of hours for which the computed $t$-statistic is greater than the critical value. This follows the approach of Narayan et al. (2016). To circumvent the limitations of the ADF test, we also use the sup-ADF test which is rolling window based and its critical values are computed using Monte Carlo simulations; see Phillips et al. (2015) for details on the procedure and Table 1 for details on critical values.

\section{B. Results}

Our results are summarized in Table 1 . To start, we refer to Panel A where full-sample results are described. The first point of note is about the sub-ADF test results reported in the last column of the table. Over the full-sample period of data, the null hypothesis of a unit root is rejected only for the $\mathrm{CAD}$, suggesting that of the four currencies only the Canadian exchange rate is characterized by explosive behavior. We compare now the explosiveness of currencies in the pre-COVID-19 and the COVID-19 samples. We find that in the pre-COVID-19 sample, none of the series displays any evidence of explosive behavior. That is, the null hypothesis of a unit root cannot be rejected at the $10 \%$ level. By comparison, we see that in the COVID-19 period the unit root null is rejected in favor of explosiveness for YEN, CAD, and GBP, suggesting that only for the EURO there is no evidence of bubbles type behavior.
The next set of results is about the intensity of bubble activity. As mentioned in Section II.A, our approach follows the literature in estimating the number of hours of bubbles in the two sub-samples of data. We start with the Canadian dollar and see that in the pre-COVID-19 period, there were 80 hours over which bubble activity was detected. In the COVID-19 sample, it increased by $26.25 \%$ to 101 hours. For the EURO, bubble activity jumped from 99 hours (preCOVID-19) to 134 hours (COVID-19 sample)-a 35.35\% increase in bubble activity. In the case of the GBP, the corresponding increase was from 115 hours to 170 hours, representing a growth in bubble activity of $47.83 \%$. Finally, we notice that the Japanese Yen saw the largest jump in bubble activity, from 97 hours in the pre-COVID-19 period to 241 hours in the COVID-19 period. This amounts to a $148.45 \%$ increase in bubble activity.

We also estimate and report the number of continuous hours of bubble activity in both periods. Except for the CAD, all currencies have seen greater number of continuous hours of bubble activity in the COVID-19 period compared to the pre-COVID-19 period. Again, the Yen sees the largest jump in bubble activity.

\section{Concluding remarks}

In this note we start on exploring bubble activity in exchange rates motivated by the broader implications of the COVID-19 pandemic. Using four currencies (CAD, YEN, EURO, and GBP) sampled at hourly frequency, we show that in the pre-COVID-19 period (July 2019 to Dec 2019) there is no evidence of explosiveness in exchange rates except for CAD. In the COVID-19 sample (Jan 2020 to Sept 2020), we find stronger evidence of exchange rate explosiveness: except for the EURO, all currencies are characterized by explosive behavior. In terms of bubble activity, we find greater number of hours of bubble activity in the COVID-19 period compared to the pre-COVID-19 sample. We conclude that the intensity of bubble activity has increased in the COVID-19 period, implying that on average the exchange rate market has become more inefficient in the pandemic than it was prior to it.

Submitted: October 13, 2020 AEDT, Accepted: October 21, 2020 AEDT 
Table 1: Bubbles test results

Panel A: Full-sample period (01/07/2019: 2:00am to 04/09/2020: 5:00pm), $T=5270$

\begin{tabular}{|c|c|c|c|}
\hline & No. of hours of bubbles & Longest continuous period of bubbles & SDAF test \\
\hline YEN & 338 & 37 & 0.537 \\
\hline CAD & 190 & 46 & $5.917^{* * *}$ \\
\hline EURO & 236 & 41 & -0.191 \\
\hline GBP & 297 & 42 & 1.160 \\
\hline \multicolumn{4}{|c|}{ Panel B: Pre-COVID-19 sample period (01/07/2019: 2:00am to 31/12/2019: 5:00pm), T=2244 } \\
\hline & No. of hours of bubbles & Longest continuous period of bubbles & SDAF test \\
\hline YEN & 97 & 22 & 0.537 \\
\hline CAD & 80 & 21 & -0.204 \\
\hline EURO & 99 & 16 & -0.606 \\
\hline GBP & 115 & 38 & 1.160 \\
\hline \multicolumn{4}{|c|}{ Panel C: COVID-19 sample period (01/07/2019: 2:00am to 31/12/2019: 5:00pm), $T=3026$} \\
\hline & No. of hours of bubbles & Longest continuous period of bubbles & SDAF test \\
\hline YEN & 241 & 37 & $3.068^{* * *}$ \\
\hline CAD & 101 & 14 & $4.577^{* * *}$ \\
\hline EURO & 134 & 17 & 0.753 \\
\hline GBP & 170 & 42 & $5.737^{* * *}$ \\
\hline \multicolumn{4}{|c|}{ Panel D: Critical values } \\
\hline Sample size, $T$ & $1 \%$ & $5 \%$ & $10 \%$ \\
\hline$T=5270$ & 2.343 & 1.639 & 1.393 \\
\hline$T=2244$ & 2.328 & 1.611 & 1.271 \\
\hline$T=3026$ & 2.037 & 1.569 & 1.316 \\
\hline
\end{tabular}

This table reports the bubbles test results for the four currencies, namely, the Canadian dollar (CAD), the Japanese Yen (YEN), the European Euro (EURO), and the British pound (GBP). The data sample is from 01/07/2019: 2:00am to 04/09/2020: 5:00pm. The full-sample of data is split into a pre-COVID-19 (01/07/2019: 2:00am to 31/12/2019: 5:00pm) and the COVID-19 sample (1/01/2020: 1:00am to 04/09/2-2-: 5:00pm). The null hypothesis is that there is a unit root, which is tested against the alternative hypothesis of an explosive root. The idea is based on Phillips et al. (2011), where an augmented Dickey-Fuller (ADF) model is estimated using least squares. We estimate the ADF model recursively using subsets of the sample data. The corresponding $t$-test statistics are generated following the procedure outlined in Phillips et al. (2011). We set the initial window to 100 hours. The critical values used for testing the null hypothesis are given $\operatorname{byCV}(s)=\frac{\log (\log (n s))}{100}$. By generating these $t$-statistics and comparing them with the critical value, we obtain the number of hours for which the computed $t$-statistic is greater than the critical value (column 2) and the longest continuous hourly period of bubbles (column 3 ). The critical values for the sup-ADF test (last column) are generated using Monte Carlo simulations using 500 replications specific to the three samples of data. The critical values at the $1 \%, 5 \%$ and $10 \%$ levels are presented in Panel D. Finally, ${ }^{* * * *}$ denotes statistical significance at the $1 \%$ level-that is, the null hypothesis of a unit root is rejected in favor of an explosive series. 


\section{REFERENCES}

Al-Awadhi, A. M., Al-Saifi, K., Al-Awadhi, A., \& Alhammadi, S. (2020). Death and contagious infectious diseases: Impact of the COVID-19 virus on stock market returns. Journal of Behavioral and Experimental Finance, 27, 100326. https://doi.org/1 $\underline{0.1016 / j . j b e f .2020 .100326}$

Bettendorf, T., \& Chen, W. (2013). Are there bubbles in the Sterling-dollar exchange rate? New evidence from sequential ADF tests. Economics Letters, 120(2), 350-353. https://doi.org/10.1016/j.econlet.2013.04.03 $\underline{9}$

Devpura, N., \& Narayan, P. K. (2020). Hourly oil price volatility: The role of COVID-19. Energy Research Letters, 1(2), 13683. https://doi.org/10.46557/001c.13 $\underline{683}$

Ertuğrul, H. M., Güngör, B. O., \& Soytaş, U. (2020). The Effect of the COVID-19 Outbreak on the Turkish Diesel Consumption Volatility Dynamics. Energy Research Letters. https://doi.org/10.46557/001c.1749 $\underline{6}$

Fu, M., \& Shen, H. (2020). COVID-19 and corporate performance in the energy industry. Energy Research Letters, 1(1), 12967. https://doi.org/10.46557/001c.12 $\underline{967}$

Garber, P. M. (1989). Tulipmania. Journal of Political Economy, 97(3), 535-560. https://doi.org/10.1086/26 $\underline{1615}$

Gil-Alana, L. A., \& Monge, M. (2020). Crude oil prices and COVID-19: Persistence of the shock. Energy Research Letters, 1(1), 13200. https://doi.org/10.4655 7/001c.13200

Gu, X., Ying, S., Zhang, W., \& Tao, Y. (2020). How do firms respond to COVID-19? First evidence from Suzhou, China. Emerging Markets Finance and Trade, 56(10), 2181-2197. https://doi.org/10.1080/1540496 $\underline{\mathrm{X} .2020 .1789455}$

Haroon, O., \& Rizvi, S. A. R. (2020a). Flatten the curve and stock market liquidity-An Inquiry into emerging economies. Emerging Markets Finance and Trade, 56(10), 2151-2161. https://doi.org/10.1080/15 40496x.2020.1784716

Haroon, O., \& Rizvi, S. A. R. (2020b). COVID-19: Media coverage and financial markets behavior-A sectoral inquiry. Journal of Behavioral and Experimental Finance, 27, 100343. https://doi.org/1 $\underline{0.1016 / j . j b e f .2020 .100343}$
Hu, Y., \& Oxley, L. (2017). Are there bubbles in exchange rates? Some new evidence from G10 and emerging market economies. Economic Modelling, 64, 419-442. https://doi.org/10.1016/j.econmod.201 7.02.022

Huang, W., \& Zheng, Y. (2020). COVID-19: Structural changes in the relationship between investor sentiment and crude oil futures price. Energy Research Letters, 1(2), 13685. https://doi.org/10.4655 7/001c.13685

Iyke, B. N. (2020a). COVID-19: The reaction of US oil and gas producers to the pandemic. Energy Research Letters, 1(2), 13912. https://doi.org/10.46557/001c.13 $\underline{912}$

Iyke, B. N. (2020b). The disease outbreak channel of exchange rate return predictability: Evidence from COVID-19. Emerging Markets Finance and Trade, 56(10), 2277-2297. https://doi.org/10.1080/1540496 $\underline{\mathrm{x} .2020 .1784718}$

Liu, L., Wang, E.-Z., \& Lee, C.-C. (2020). Impact of the COVID-19 pandemic on the crude oil and stock markets in the US: A time-varying analysis. Energy Research Letters, 1(1), 13154. https://doi.org/10.4655 7/001c.13154

Narayan, P. K. (2020). Oil price news and COVID-19-Is there any connection? Energy Research Letters, 1(1), 13176. https://doi.org/10.46557/001c.13176

Narayan, P. K., Devpura, N., \& Wang, H. (2020). Japanese currency and stock market-What happened during the COVID-19 pandemic? Economic Analysis and Policy, 68, 191-198. https://doi.org/10.1016/j.ea p.2020.09.014

Narayan, P. K., Sharma, S. S., \& Phan, D. H. B. (2016). Asset price bubbles and economic welfare.

International Review of Financial Analysis, 44, 139-148. https://doi.org/10.1016/i.irfa.2016.01.011

Phillips, P. C. B., Shi, S., \& Yu, J. (2015). Testing for multiple bubbles: Historical episodes of exuberance and collapse in the S\&P 500. International Economic Review, 56(4), 1043-1078. https://doi.org/10.1111/ier e.12132

Phillips, P. C. B., Wu, Y., \& Yu, J. (2011). Explosive behavior in the 1990s NASDAQ: When did exuberance escalate asset values? International Economic Review, 52(1), 201-226. https://doi.org/10.1111/j.146 8-2354.2010.00625.x 
Qin, M., Zhang, Y.-C., \& Su, C.-W. (2020). The Essential Role of Pandemics: A Fresh Insight into the Oil Market. Energy Research Letters, 1(1). https://do i.org/10.46557/001c.13166

Salisu, A., \& Adediran, I. (2020). Uncertainty due to infectious diseases and energy market volatility. Energy Research Letters, 1(2), 14185. https://doi.org/ 10.46557/001c.14185

Salisu, A.A., Akanni, L., \& Raheem, I. (2020). The COVID-19 global fear index and the predictability of commodity price returns. Journal of Behavioral and Experimental Finance, 27, 100383.

Salisu, Afees A., \& Vo, X. V. (2020). Predicting stock returns in the presence of COVID-19 pandemic: The role of health news. International Review of Financial Analysis, 71, 101546. https://doi.org/10.1016/j.irfa.20 20.101546
Sha, Y., \& Sharma, S. S. (2020). Research on Pandemics Special Issue of the Journal Emerging Markets Finance and Trade. Emerging Markets Finance and Trade, 56(10), 2133-2137. https://doi.or $\mathrm{g} / 10.1080 / 1540496 x .2020 .1795467$

Xiong, H., Wu, Z., Hou, F., \& Zhang, J. (2020). Which firm-specific characteristics affect the market reaction of Chinese listed companies to the COVID-19 pandemic? Emerging Markets Finance and Trade, 56(10), 2231-2242. https://doi.org/10.1080/15 40496x.2020.1787151

Zaremba, A., Kizys, R., Aharon, D. Y., \& Demir, E. (2020). Infected markets: Novel coronavirus, government interventions, and stock return volatility around the globe. Financial Research Letters, 35, 101597. https://doi.org/10.1016/i.frl.2020.101597

Zhang, D., Hu, M., \& Ji, O. (2020). Financial markets under the global pandemic of COVID-19. Finance Research Letters, 36, 101528. https://doi.org/10.1016/ j.frl.2020.101528 